\title{
Copépodes e isópodes parasitos de nove espécies de peixes marinhos (Osteichthyes) do litoral do Estado do Rio de Janeiro, Brasil
}

\section{Parasitic copepods and isopods on nine species of marine fishes (Osteichthyes) from the coast of Rio de Janeiro State, Brazil}

\author{
${ }^{1}$ Naraiana Lopes Taborda \\ 1 Julia Ramos de Lima \\ ${ }^{1}$ Viviane da Silva Pinheiro \\ ${ }^{2}$ Dimitri Ramos Alves \\ ${ }^{3}$ André Resende Senna
}

1 Discente do Curso de Ciências Biológicas do Centro Universitário de Volta Redonda - UniFOA

2 Docente do Curso de Ciências Biológicas do Centro Universitário de Volta Redonda - UniFOA / dimitri.alves@foa.org.br

3 Universidade do Estado do Rio de Janeiro (UERJ). Faculdade de Formação de Professores (FFP), Departamento de Ciências

\section{RESUMO}

Entre fevereiro de 2009 a junho de 2012, 368 espécimes de nove espécies de peixes marinhos (Osteichthyes) coletados ao longo da costa do Estado do Rio de Janeiro $\left(21^{\circ}-23^{\circ} \mathrm{S}, 41^{\circ}-45^{\circ} \mathrm{W}\right)$ foram necropsiados para estudar sua fauna parasita de Copepoda e Isopoda. Foram identificadas 11 espécies de copépodes e três espécies de isópodes parasitos. Sendo coletados 852 espécimes de copépodes e quatro de isópodes. 0 mais prevalente e abundante dentre os crustáceos parasitos foi Taeniacanthus lagocephali Pearse, 1952, enquanto Coryphaena hippurus Linnaeus, 1758, foi a espécie de hospedeiro com a maior diversidade de espécies de ectoparasitos. Os gêneros Ceratothoa Dana, 1852, e Mothocya Hope, 1851, são registrados pela primeira vez no Brasil neste estudo.

\section{PALAVRAS-CHAVE}

Ectoparasitos; Copepoda: Isopoda: Peixes marinhos.

\section{ABSTRACT}

From February 2009 and June 2012, 368 specimens of nine species of marine fishes (Osteichthyes) collected from coast of the State of Rio de Janeiro $\left(21^{\circ}-23^{\circ} \mathrm{S}\right.$, $41^{\circ}-45^{\circ} \mathrm{W}$ ), were necropsied to study their copepod and isopod parasite fauna. Were identified 11 species of Copepoda and three species of Isopoda parasites. Eight hundred fifty-two specimens of copepod and four of isopod were collected. The most prevalent and abundant among the parasite crustaceans was Taeniacanthus lagocephali Pearse, 1952, while Coryphaena hippurus Linnaeus, 1758 was the fish species with the greatest diversity of ectoparasites species. The genera Ceratothoa Dana, 1852 and Mothocya Hope, 1851 are recorded for the first time from Brazil in this study.

\section{KEYWORDS}

Ectoparasites; Copepoda; Isopoda; Marine fishes.

\section{Como você deve citar?}

TABORDA, N. L. et al. Copépodes e isópodes parasitos de nove espécies de peixes marinhos (Osteichthyes) do litoral do Estado do Rio de Janeiro, Brasil. Cadernos UniFOA, Volta Redonda, n 25 , quadrimestral, p. 105-111, ago. 2014. 


\section{INTRODUÇÃO}

Os peixes marinhos constituem, em nível mundial, um dos recursos alimentares de maior qualidade e abundância; consequentemente, esse grupo de vertebrados vem sendo alvo de numerosas pesquisas relacionadas a seus mais diversos aspectos biológicos. Entretanto, o aspecto da influência do parasitismo na biologia dos peixes marinhos tem sido relativamente pouco estudado (LUQUE, 2004a, b). Os ectoparasitos são componentes frequentes nas comunidades de peixes marinhos da Região Neotropical. Basicamente, esse grupo de parasitos é formado por monogenéticos (Monogenea), copépodes (Copepoda), branquiúros (Branchiura), isópodes (Isopoda) e sanguessugas (Hirudinea) (ROHDE et al., 1995; LUQUE et al., 2004; LUQUE; POULIN, 2007; POULIN et al., 2011). Entretanto, dos táxons citados, apenas os monogenéticos e copépodes têm sido relativamente estudados (KOHN; COHEN, 1998; LUQUE et al., 1998; KOHN; PAIVA, 2000; ALVES et al., 2003; LUQUE; ALVES, 2003; THATCHER; PEREIRA-JUNIOR, 2004; KOHN et al., 2006; LUQUE; TAVARES, 2007; CAVALCANTI et al., 2013). No Brasil, os estudos mais abrangentes realizados com copépodes parasitos de peixes marinhos foram realizados por Boxshall e Montú (1997), Ho (1998a, b), Luque e Tavares (2007) e Boos et al. (2012). Os estudos referentes aos isópodes são escassos no Brasil. No presente trabalho, apresentamos uma lista de 11 espécies de copépodes e três de isópodes, associados a nove espécies de peixes marinhos ao longo da costa do Estado do Rio de Janeiro.

\section{MATERIAIS E MÉTODOS}

Entre fevereiro de 2009 e junho de 2012, foram necropsiados 368 espécimes de peixes distribuídos nas seguintes espécies: Coryphaena hippurus Linnaeus, 1758 (Perciformes: Coryphaenidae) $(\mathrm{n}=68)$; Cynoscion guatucupa (Cuvier, 1830) $(n=25)$, Larimus breviceps Cuvier, $1830(n=17)$ e Micropogonias furnieri (Desmarest, 1823) $(\mathrm{n}=01)$ (Perciformes: Sciaenidae); Lagocephalus laevigatus (Linnaeus, 1766) ( $=70)$ e L. lagocephalus (Linnaeus, 1758) $(n=37)$ (Tetraodontiformes: Tetraodontidae); Merluccius hubbsi Marini, 1933 (Gadiformes: Merlucciidae) $(n=31)$; Thyrsitops lepidopoides (Cuvier, 1832) (Perciformes: Gempylidae) $(n=55)$ e Trachurus lathami Nichols, 1920 (Perciformes: Carangidae) $(n=64)$. A determinação dos hospedeiros foi feita de acordo com Fiqueiredo (1978, 2000); Menezes e Figueiredo (1980, 1985), Figueiredo et al. (2002) e Nelson (2006). Os peixes foram adquiridos de pescadores artesanais que atuam no litoral do Estado do Rio de Janeiro. Uma vez obtidos, os mesmos foram acondicionados em caixas de isopor contendo gelo, para assegurar boas condições para a coleta dos parasitos e protegê-los durante o transporte até o Laboratório de Zoologia do Centro Universitário de Volta Redonda (UniFOA). Os copépodes e isópodes foram coletados na superfície corporal, fossas nasais, cavidade branquial, cavidade bucal e brânquias, sendo posteriormente fixados e conservados em etanol $70^{\circ} \mathrm{GL}$. Para identificação, os espécimes foram clarificados em ácido láctico e montados em lâminas. A coleta, registro e processamento dos parasitos foram realizados de acordo com os procedimentos indicados por Eiras et al. (2000).

\section{RESULTADOS}

Dentre os espécimes de peixes necropsiados, foram identificadas 11 espécies de copépodes e três de isópodes parasitos: Bomolochus sp. (Poecilostomatoida: Bomolochidae); C. balistae Steenstrup e Lütken, 1861; Caligus bonito Wilson, 1905 e Caligus sp. (Siphonostomatoida: Caligidae); Charopinopsis sp. (Siphonostomatoida: Lernaeopodidae); Chondracanthus merlucci (Holten, 1802) (Poecilostomatoida: Chondracanthidae); Euryphorus brachypterus (Gerstaecker, 1853) e E. nordmanni Milne Edwards, 1840 (Siphonostomatoida: Caligidae); Lernanthropus cynoscicola Timi e Etchegoin, 1996 (Siphonostomatoida: Lernanthropidae); Pennella sp. (Siphonostomatoida: Pennellidae); Taeniacanthus lagocephali Pearse, 
1952 (Poecilostomatoida: Taeniacanthidae); Anilocra sp.; Ceratothoa sp. e Mothocya sp. (Isopoda: Cymothoidae). Foram coletados 852 espécimes de copépodes e quatro de isópodes. Os copépodes estão representados por oito gêneros pertencentes e sete famílias. No caso dos isópodes, são três gêneros representados por apenas uma família (Cymothoidae). Taeniacanthus lagocephali, parasitando L. lagocephalus, e L. laevigatus, foi a espécie mais prevalente e abundante. 0 dourado, Coryphaena hippurus, apresentou o maior número de ectoparasitos $(n=6)$ (Tabela 1).

Tabela 1 - Copépodes e isópodes parasitos de nove espécies de peixes marinhos (Osteichthyes) do litoral do Estado do Rio de Janeiro, Brasil.

\begin{tabular}{|c|c|c|c|c|}
\hline Parasitos & $\begin{array}{l}\text { Prevalência } \\
\text { (\%) }\end{array}$ & $\begin{array}{l}\text { Intensidade } \\
\text { Média }\end{array}$ & $\begin{array}{l}\text { Abundância } \\
\text { Média }\end{array}$ & Hospedeiro \\
\hline \multicolumn{5}{|l|}{ Copepoda } \\
\hline Bomolochus sp. & 12,7 & 1 & $0,12 \pm 0,33$ & T. lepidopoides \\
\hline Caligus balistae & 1,5 & 1 & $0,01 \pm 0,12$ & C. hippurus* \\
\hline Caligus bonito & 54,4 & $3,7 \pm 2,4$ & $2 \pm 2,6$ & C. hippurus* \\
\hline Caligus sp. & 4,68 & $1,66 \pm 0,57$ & $0,7 \pm 0,36$ & T. lathami** \\
\hline Charopinopsis sp. & 14,7 & $2,6 \pm 2$ & $0,57 \pm 1,4$ & C. hippurus* \\
\hline $\begin{array}{l}\text { Chondracanthus } \\
\text { merlucci }\end{array}$ & 22,5 & $1,14 \pm 0,37$ & $0,33 \pm 0,56$ & M. hubbsi \\
\hline $\begin{array}{l}\text { Euryphorus } \\
\text { brachypterus }\end{array}$ & 22,05 & $2,4 \pm 2,3$ & $0,52 \pm 1,5$ & C. hippurus* \\
\hline Euryphorus nordmanni & 35,3 & $3,1 \pm 3,5$ & $1,1 \pm 2,5$ & C. hippurus* \\
\hline $\begin{array}{l}\text { Lernanthropus } \\
\text { cynoscicola }\end{array}$ & 12,3 & 1 & $0,1 \pm 0,4$ & C. guatucupa \\
\hline Pennella sp. & 17,6 & $1,2 \pm 0,6$ & $0,26 \pm 0,6$ & C. hippurus* \\
\hline \multirow[t]{2}{*}{$\begin{array}{l}\text { Taeniacanthus } \\
\text { lagocephali }\end{array}$} & 64,8 & $4,9 \pm 3,9$ & $3,2 \pm 3,9$ & L. lagocephalus \\
\hline & 100 & $10,7 \pm 11,2$ & $10,7 \pm 11,2$ & L. laevigatus \\
\hline \multicolumn{5}{|l|}{ Isopoda } \\
\hline Anilocra sp. & 5,8 & 1 & $0,05 \pm 0,24$ & L. breviceps \\
\hline Ceratothoa sp. & 100 & 2 & 2 & $\begin{array}{l}\text { Micropogonias } \\
\text { furnieri }\end{array}$ \\
\hline Mothocya sp. & 3,2 & 1 & $0,04 \pm 0,2$ & M. hubbsi \\
\hline
\end{tabular}

* Marques e Alves (2011); ** Gonçalves e Alves (2012)

\section{DISCUSSÃo}

No presente estudo, foram analisadas as faunas de ectoparasitos, ênfase em copépodes e isópodes, de nove espécies de peixes marinhos, distribuídos em seis famílias, do sudeste brasileiro. 
Desse grupo de hospedeiros, foram identificadas 14 espécies de ectoparasitos: 11 copépodes e três isópodes. Embora o número de espécies de ectoparasitos tenha sido maior do que a de hospedeiros, a riqueza parasitária pode ser considerada baixa, com a média de 1,2 espécies de copépodes por hospedeiros. Em relação aos isópodes, o número é menor: 0,3 espécies de isópodes por hospedeiro. $\mathrm{Na}$ região Neotropical, principalmente no Brasil, as espécies de copépodes parasitos têm sido registradas nas com frequência nas comunidades parasitárias de peixes marinhos (ROHDE et al., 1995; LUQUE, TAVARES, 2007; POULIN et al., 2011). Entretanto, segundo Luque e Tavares (2007), o conhecimento sobre as espécies de copépodes parasitos de peixes marinhos estudadas no Brasil, principalmente em relação à expressiva diversidade íctia, se encontra em um estado incipiente.

Segundo Luque e Tavares (2007), Caligidae é a família de copépode com maior diversidade em peixes marinhos, sendo representada por 47 espécies, sendo o gênero Caligus o abundante com mais de 200 espécies (BOXSHALL, MONTÚ, 1997; HO, 1998b). No presente estudo, foram identificadas cinco espécies de caligídeos: Caligus baliste, C. bonito, Caligus sp., Euryphorus brachypterus e E. nordmanni. Corroborando, dessa forma, os estudos realizados sobre copépodes parasitos de peixes marinhos da costa brasileira (BOXSHALL, MONTÚ, 1997; HO, 1998b; LUQUE, TAVARES, 2007). De acordo com Johnson et al. (2004), Caligidae é a família de copépodes mais reportada em todo o mundo, apresentando uma proporção de $61 \%$ de todas as espécies, sendo $40 \%$ dessas publicações sobre Caligus.

Ho et al. (2011) afirmaram que Lernanthropidae, seguido de Lernaeopodidae e Caligidae, é a terceira família mais ampla de parasitas de peixes, pertencente à ordem Siphonostomatoida; família esta que contem mais de 150 espécies, na qual a maioria encontra-se em águas tropicais. De acordo com Özel (2004) e a base de dados WoRMS (World Register of Marine Species) (BOXSHALL, 2013), Lernanthropus é o maior gênero da família Lernanthropidae, possuindo mais de 110 espécies. Entretanto, dentre as nove espécies de peixes aqui analisados, Lernanthropidae somente esteve representado por Lernanthropus cynoscicola, com baixa prevalência (12,3\%).

Um exemplar de Ceratothoa sp. foi encontrado em Micropogonias furnieri, um de Mothocya sp. foi encontrado em Merluccius hubbsi, e dois espécimes de Anilocra sp. foram encontrados em Coryphaena hippurus e Larimus breviceps. Os gêneros Ceratothoa e Mothocya são registrados pela primeira vez no Brasil neste estudo. As três espécies de Isopoda Cymothoidae se diferenciam das demais espécies de seus gêneros, tratando-se de espécies ainda desconhecidas para a ciência, em serão descritas em publicações subsequentes.

\section{AGRADECIMENTOS}

Um dos autores, André R. Senna, agradece à Fundação Carlos Chagas Filho de Amparo à Pesquisa do Estado do Rio de Janeiro (FAPERJ) pelo fomento cedido na forma de Auxílio Instalação (E-26/110.773/2012). Este projeto recebeu suporte financeiro do Centro Universitário de Volta Redonda (UniFOA). 


\section{REFERÊNCIAS}

ALVES, D. R.; LUQUE, J. L.; PARAGUASSÚ, A. R. Acanthochondria triangularis sp. nov. (Copepoda, Poecilostomatoida, Chondracanthidae) parasitic on Urophycis brasiliensis and U. mystaceus (Osteichthys, Phycidae) from the Southern Brazilian coastal zone. Acta Parasitologica, v. 48, p. 19 - 23, 2003.

BOOS, H.; BUCKUP, G. B.; BUCKUP, L.; ARAUJO, P. B.; MAGALHÃES, C.; ALMERÃO, M. P.; SANTOS, R. A.; MANTELATTO, F. L. Checklist of the Crustacea from the state of Santa Catarina, Brazil. Check List, v. 8, n. 6, p. 1020-1046, 2012.

BOXSHALL, G. (2013). Lernanthropidae. In: Walter, T.C. \& Boxshall, G. (2013). World of Copepods database. World Register of Marine Species. Disponível em: <http://www.marinespecies.org/aphia. php? $p=$ taxdetails\&id=135526> Acessado em: 30 de Setembro de 2013

BOXSHALL, G. A.; MONTÚ, M. Copepods parasitic on Brazilian coastal fishes: A handbook. Nauplius, v. 5, p. $1-225,1997$.

CAVALCANTI, E. T. S.; NASCIMENTO, W. S.; TAKEMOTO, R. M.; ALVES, L. C.; CHELLAPPA, S. Ocorrência de crustáceos ectoparasitos no peixe ariacó, Lutjanus synagris (Linnaeus, 1758) nas águas costeiras do Rio Grande do Norte, Brasil. Biota Amazônia, v. 3, n. 1, p. 94-99, 2013.

EIRAS, J. C.; TAKEMOTO, R. M.; PAVANELLI, G. C. Métodos de estudo e técnicas laboratoriais em parasitologia de peixes. Ed. Universidade Estadual de Maringá, Maringá. 171 p., 2000.

FIQUEIREDO, J. L. Manual de peixes mari-hos do sudeste do Brasil. VI. Teleostei (5). São Paulo, Museu de Zoologia, Univ. São Paulo, 116 p., 2000.

FIQUEIREDO, J. L. Manual de peixes marinhos do sudeste do Brasil II.Teleostei (1). São Paulo, Museu de Zoologia, Univ. São Paulo, 110p., 1978.

FIQUEIREDO, J. L.; SANTOS, A. P.; YAMAGUT, N.; BERNARDES, R. A.; ROSSI-WONGTSCHOWSKI, C. L. B. Peixes da Zona Econômica Exclusiva da Região Sudeste-Sul do Brasil. 244 p., 2002.

GONÇALVES, P. H. S.; ALVES, D. R. Ecologia da comunidade de metazoários parasitos do xixarro, Trachurus lathami Nichols, 1920 (Osteichthyes: Carangidae) do litoral do Estado do Rio de Janeiro, Brasil. Cardernos UniFOA, v. 20, p. 105-113, 2012.

HO, J. S. Maxillopoda - Copepoda. Poecilostomatoida. Marine Parasites. In: Young, P. s. (ed.) Catalogue of Crustacea of Brazil. Rio de Janeiro: Museu Nacional, Série Livros v. 6, p. 235-240, 1998a.

HO, J. S. Maxillopoda - Copepoda. Siphonostomatoida. In: Young, P. s. (ed.) Catalogue of Crustacea of Brazil. Rio de Janeiro: Museu Nacional, Série Livros v. 6, p. 251-260, 1998b.

HO, J.S.; LIU, W. C.; LIN, C. L. Six Species of the Lernanthropidae (Crustacea: Copepoda) Parasitic on Marine Fishes of Taiwan, with a Key to 18 Species of the Family Known from Taiwan. Zoological Studies, v. 50, n. 5, p.611-635, 2011.

JOHNSON, S. C.; TREASURER, J. W.; BRAVO, S.; NAGASAWA, K.; KABATA, Z. A Review of the Impact of Parasitic Copepods on Marine Aquaculture. Zoological Studies, v. 43, n.2, p.229-243, 2004. 
KOHN, A.; PAIVA, M. P. Fishes parasitised by Monogenea in South America. In G Salgado-Maldonado, AN García-Aldrete, VM Vidal-Martínez (eds), Metazoan Parasites in the Neotropics: A Systematic and Ecological Respective, Instituto de Biologia, UNAM, p. 25-60, 2000.

KOHN, A.; COHEN, S. C. South American Monogenea: list of species, hosts and geographical distribution. International Journal for Parasitology, v. 28, n. 10, p. 1517-1554, 1998.

KOHN, A.; COHEN, S. C.; SALGADO-MALDONADO, G. Checklist of Monogenea parasites of freshwater and marine fishes, amphibians and reptiles from Mexico, Central America and Caribbean. Zootaxa, v. 1289, p. 1-114, 2006.

LUQUE, J. L.; MOUILLOT, D.; POULIN, R. Parasite biodiversity and its determinants in coastal marine teleost fishes of Brazil. Parasitology, v. 128, p. 671-682, 2004.

LUQUE, J. L.; POULIN, R. Metazoan parasite species richness in Neotropical fishes: hotspots and the geography of biodiversity. Parasitology, v. 134, p. 865-878, 2007.

LUQUE, J. L. Biologia, epidemiologia e controle de parasitos de peixes. Revista Brasileira de Parasitologia Veterinária. v. 13, n. 1, p. 1-5, 2004b.

LUQUE, J. L.; ALVES, D. R. Pseudolernentoma brasiliensis n. g., n. sp. (Copepoda: Poecilostomatoida: Chondracanthidae) parasitic on Genypterus brasiliensis (Osteichthyes, Ophidiidae) from off the state of Rio de Janeiro, Brazil. Systematic Parasitology, v. 56, p. 195-199, 2003.

LUQUE, J. L.; CHAVES, N. D.; CEZAR, A. D. Novos registros de copépodes caligóides parasitos de peixes marinhos do Brasil. Nauplius, v. 6, p. 9-16, 1998.

LUQUE, J. L.; TAVARES, L. E. R. Checklist of Copepoda associated with fishes from Brazil. Zootaxa, v. 1579, p. 1-39, 2007.

LUQUE, J. L.; TAVARES, L. E. R. Checklist of Copepoda associated with fishes from Brazil. Zootaxa, v. 1579, p. 1-39, 2007

LUQUE, J. L. Parasitologia de peixes marinhos na América do Sul: estado atual e perspectivas. In: M. J. T. R. PAIVA; R. M. TAKEMOTO; M. L. A. P. LIZAMA. (Org.). Sanidade de organismos aquáticos. São Paulo: Varela, v. 1, p. 199-215, 2004a.

MARQUES, L. C.; ALVES, D. R. Ecologia da comunidade de metazoários parasitos do dourado, Coryphaena hippurus Linnaeus, 1758, (Osteichthyes: Coryphaenidae) do litoral do Estado do Rio de Janeiro, Brasil. Cardernos UniFOA, v. 16, p. 111 - 122, 2011.

MENEZES, N. A.; FIGUEIREDO, J. L. Manual de Peixes Marinhos do Sudeste do Brasil. IV. Teleostei (3). Museu de Zoologia, Universidade de São Paulo, São Paulo, 96 p., 1980.

MENEZES, N. A.; FIGUEIREDO, J. L. Manual de peixes marinhos do sudeste do Brasil. V Teleostei (4). São Paulo, Museu de Zoologia, Univ. São Paulo, 105p., 1985.

NELSON, J. S. Fishes of the world. 4. ed. Editora Wiley, New Jersey, USA. 2006. 
ÖZEL, I., ÖKTENER, A.; AKER, V. A Morphological Study (SEM) on a Parasitic Copepod: Lernanthropus kroyeri van Beneden, 1851. E.U. Journal of Fisheries \& Aquatic Sciences. v.21, n. 3-4, p. 335-337, 2004.

POULIN, R.; GUILHAUMON, F.; RANDHAWA, H. S.; LUQUE, J. L.; MOUILLOT, D. Identifying hotspots of parasite diversity from species-area relationships: host phylogeny versus host ecology. Oikos, v. 120, p. $740-747,2011$.

ROHDE, K.; HAYWARD, C.; HEAP, M. Aspects of the ecology of metazoan ectoparasites of marine fishes. International Journal for Parasitology, v. 25, n 8, p. 945-970, 1995.

THATCHER, V. E.; PEREIRA-JUNIOR, J.. Brasilochondria riograndensis gen. nov., sp. nov. (Copepoda, Chondracanthidae) a parasite of flounders of Rio Grande do Sul State, Brazil. Revista Brasileira de Zoologia, v. 21, n.3, p. $515-518,2004$. 